\title{
Generalized Moment Generating Functions of Random Variables and Their Probability Density Functions
}

\author{
Matthew Chukwuma Michael, ${ }^{1, *}$, Oyeka Cyprain Anene ${ }^{2}$, Ashinze Mpuruoma Akudo ${ }^{1}$, Igabari John Nwabueze ${ }^{3}$ \\ ${ }^{1}$ Department of Mathematics and Statistics, School of Applied Sciences, Delta State Polytechnic, Ogwashi-Uku \\ ${ }^{2}$ Department of Statistics, Faculty of Physical Sciences, Nnamdi Azikiwe University, Awka, Anambra State \\ ${ }^{3}$ Department of Mathematics, Delta State University, Abraka, Delta State \\ *Corresponding author: megawaves4life@yahoo.com
}

\begin{abstract}
This paper seeks to develop a generalized method of generating the moments of random variables and their probability distributions. The Generalized Moment Generating Function is developed from the existing theory of moment generating function as the expected value of powers of the exponential constant. The methods were illustrated with the Beta and Gamma Family of Distributions and the Normal Distribution. The methods were found to be able to generate moments of powers of random variables enabling the generation of moments of not only integer powers but also real positive and negative powers. Unlike the traditional moment generating function, the generalized moment generating function has the ability to generate central moments and always exists for all continuous distribution but has not been developed for any discrete distribution.
\end{abstract}

Keywords: generalized, moments, generating, functions, distribution function, arbitrary constant

Cite This Article: Matthew Chukwuma Michael, Oyeka Cyprain Anene, and Ashinze Mpuruoma Akudo, "Generalized Moment Generating Functions of Random Variables and Their Probability Density Functions." American Journal of Applied Mathematics and Statistics, vol. 5, no. 2 (2017): 49-53. doi: 10.12691/ajams-5-2-2.

\section{Introduction}

The $n^{\text {th }}$ moment of a random variable, $X$, about an arbitrarily chosen constant, $\lambda$, is defined as the expected value of the $n^{\text {th }}$ power of the difference between the random variable, $X$, and the arbitrarily chosen constant, $\lambda$. If $\lambda$ is equal to zero the moment of interest is called the non-central moment or moment about zero; however, if the constant, $\lambda$, is equal to $\mu$, the mean of the distribution, interest is on the central moments $[1,2,3]$.

The basic method of determining the $n^{\text {th }}$ non-central moment of a random variable, $X$, is the moment generating function (here referred to as the traditional moment generating function), defined as $M_{X}(t)=E\left(e^{t x}\right)$. $[4,5] . M_{X}(t)$ is usually difficult to evaluate and may not exist for some distributions.

We propose in this paper to develop a more versatile, easier and quicker to apply function which for lack of better nomenclature shall be called the generalized moment generating function. The method, which is similar to the traditional moment generating function in formation is able to generate moments of powers of random variables that are not necessarily positive integers but may be any real number.

\section{The Generalized Moment Generating Function}

Let $X$ ba a random variable whose $p d f$ is denoted by $f(x) ; c$ is any real number that does not need to be positive or integral and $\lambda$ is any arbitrarily chosen constant Let $G_{X}(t)$ define the generalized moment generating function of $X$. Then,

$$
G_{X}(t)=E\left[e^{t\left(X^{c}+\lambda\right)}\right]
$$

$G_{X}(t)$, Equation 1, may be evaluated as follows:

$$
\begin{aligned}
& G_{X}(t)=E\left[e^{t\left(X^{c}+\lambda\right)}\right]=E\left[\sum_{n=0}^{\infty} \frac{\left[t\left(X^{c}+\lambda\right)\right]^{n}}{n !}\right] \\
& =\sum_{n=0}^{\infty} E\left(X^{c}+\lambda\right)^{n} \cdot \frac{t^{n}}{n !} \\
& \therefore G_{X}(t)=E\left[\begin{array}{l}
\left(X^{c}+\lambda\right)^{0} \frac{t^{0}}{0 !}+\left(X^{c}+\lambda\right)^{1} \frac{t^{1}}{1 !}+\ldots \\
+\left(X^{c}+\lambda\right)^{n} \frac{t^{n}}{n !}+\ldots
\end{array}\right]
\end{aligned}
$$

The coefficient of $\frac{t^{n}}{n !}$ In Equation 2 gives the $n^{t h}$ generalized moment of $X$. That is,

$$
\begin{gathered}
G_{n}(c ; \lambda)=E\left[X^{c}+\lambda\right]^{n}=E\left[\sum_{r=0}^{n}\left(\begin{array}{l}
n \\
r
\end{array}\right) \lambda^{n-r} X^{c r}\right] \\
\therefore G_{n}(c ; \lambda)=\sum_{r=0}^{n}\left(\begin{array}{l}
n \\
r
\end{array}\right) \lambda^{n-r} E\left(X^{c r}\right)
\end{gathered}
$$


Equation 3 yields the $n^{\text {th }}$ generalized moment of the random variable, $X . E\left(X^{c r}\right)$ is the non-central moment or the generalized moment about zero of the random variable, $X$.

\section{General Application}

Suppose $f(x)=2 x$ for the random variable, $X ; 0<$ $x<1$. Using Equation 3,

$$
\begin{aligned}
G_{n}(c ; \lambda) & =\sum_{r=0}^{n}\left(\begin{array}{l}
n \\
r
\end{array}\right) \lambda^{n-r} \int_{0}^{1} X^{c r} f(x) d x \\
& =\sum_{r=0}^{n}\left(\begin{array}{l}
n \\
r
\end{array}\right) \lambda^{n-r} 2 \int_{0}^{1} x^{c r+1} d x .
\end{aligned}
$$

Now,

$$
G_{n}(c ; \lambda)=\sum_{r=0}^{n}\left(\begin{array}{l}
n \\
r
\end{array}\right) \lambda^{n-r} \frac{2}{c r+2} .
$$

Hence, for $c=1, n=1$, the first moment of $X$ about $\lambda$ for the distribution is

$$
G_{1}(1 ; \lambda)=\lambda+\frac{2}{3}
$$

The first moment of the random variable, $X$, about $\lambda$.

Now, if $\lambda=0$, the first non-central moment becomes

$$
E(X)=\frac{2}{3}=\mu_{1}^{\prime}(1)=\mu_{1}(1) \text {. }
$$

The second generalized moment may be obtained using Equation 4 as

$$
G_{2}(1 ; \lambda)=\lambda^{2}+\frac{4}{3} \lambda+\frac{1}{2}
$$

Hence for $\lambda=\frac{-2}{3}$,

$$
G_{2}\left(1 ;-\frac{2}{3}\right)=\frac{1}{18}=\sigma^{2} \text {. }
$$

Equation 8 gives the variance of the random variable, $X$ for the distribution $2 x ; 0<x<1$. The same result would be obtained using classical methods.

Now, suppose $c=\frac{1}{2}$ then from Equation 4,

$$
G_{n}\left(\frac{1}{2} ; \lambda\right)=\sum_{r=0}^{n}\left(\begin{array}{l}
n \\
r
\end{array}\right) \lambda^{n} \frac{2}{\frac{r}{2}+2}
$$

Hence the first generalized moment of the distribution of $X^{\frac{1}{2}}$ becomes

$$
G_{1}\left(\frac{1}{2} ; \lambda\right)=\lambda+\frac{8}{5}
$$

Now if $\lambda=0$, the first non-central moment of the distribution of $X^{\frac{1}{2}}$ becomes,

$$
G_{1}\left(\frac{1}{2} ; 0\right)=\mu_{i}^{\prime}(1)=\mu_{1}(1)=\frac{8}{5} .
$$

The second generalized moment of the distribution of $X^{\frac{1}{2}}$ becomes

$$
G_{2}\left(\frac{1}{2} ; \lambda\right)=2 \lambda^{2}+\frac{8}{5} \lambda+2
$$

Now if $\lambda=\left(-\frac{8}{5}\right)$, the second central moment of the distribution of $X^{\frac{1}{2}}$ becomes

$$
G_{2}\left(\frac{1}{2} ;-\frac{8}{5}\right)=\operatorname{Var}\left(X^{\frac{1}{2}}\right)=2 \text {. }
$$

Hence the variance of the distribution of $X^{\frac{1}{2}}$ is 2 .

Suppose $c=-\frac{3}{2}$, then from Equation 4 , the generalized $n^{\text {th }}$ moment of the distribution of $X^{-\frac{3}{2}}$ becomes

$$
G_{n}\left(-\frac{3}{2} ; \lambda\right)=\sum_{r=0}^{n}\left(\begin{array}{l}
n \\
r
\end{array}\right) \lambda^{n-r} \frac{2}{-\frac{3 r}{2}+2}
$$

Hence, the first generalized moment is given as

$$
G_{1}\left(-\frac{3}{2} ; \lambda\right)=\lambda+4 \text {. }
$$

Thus if $\lambda=0$, the first non-central moment becomes

$$
\mu_{1}^{\prime}(1)=\mu_{1}(1)=4 \text {. }
$$

Also, the second generalized moment of the distribution is

$$
G_{2}\left(-\frac{3}{2} ; \lambda\right)=\lambda^{2}+8 \lambda-2 .
$$

Hence the second central moment is

$$
G_{2}\left(-\frac{3}{2} ;-4\right)=\operatorname{Var}\left(X^{-\frac{3}{2}}\right)=46
$$

\section{Application to Some Common Continuous Distributions}

\subsection{The Beta Family of Distributions}

A random variable $X$ is said to have a beta distribution if its density function is of the form:

$$
\begin{aligned}
& f(x)=\frac{\Gamma(\alpha+\beta)}{\Gamma(\alpha) \Gamma(\beta)} x^{\alpha-1}(1-x)^{\beta-1} \\
& 0<x<1, \alpha>0, \beta>0 .
\end{aligned}
$$


Now, using Equation 3 in Equation 2, the coefficient of $\frac{t^{n}}{n !}$ in $G_{X}(t)$ becomes

$$
\begin{aligned}
& G_{n}(c ; \lambda) \\
& =\sum_{r=0}^{n}\left(\begin{array}{l}
n \\
r
\end{array}\right) \lambda^{n-r} \frac{\Gamma(\alpha+\beta)}{\Gamma(\alpha) \Gamma(\beta)} \int_{0}^{1} X^{(c r+\alpha-1)}(1-x)^{\beta-1} d x \\
& \therefore G_{n}(c ; \lambda)=\sum_{r=0}^{n}\left(\begin{array}{l}
n \\
r
\end{array}\right) \lambda^{n-r} \frac{\Gamma(\alpha+\beta)}{\Gamma(\alpha)} \frac{\Gamma(c r+\alpha)}{\Gamma(c r+\alpha+\beta)}
\end{aligned}
$$

Hence,

$$
G_{1}(c ; \lambda)=\lambda+\frac{\Gamma(\alpha+\beta)}{\Gamma(\alpha)} \frac{\Gamma(c+\alpha)}{\Gamma(c+\alpha+\beta)} .
$$

If $c=1$, interest is on the first moment of $X$ about $\lambda$ then it yields

$$
G_{1}(1 ; \lambda)=\lambda+\frac{\alpha}{\alpha+\beta}
$$

So that if $\lambda=0$

$$
G_{1}(1 ; 0)=\mu_{1}(1)=\mu=\frac{\alpha}{\alpha+\beta} .
$$

This is the first moment about zero, mean, of the Beta distribution.

Suppose $n=2$ and $c=1$ then, from Equation 20

$$
G_{2}(1 ; \lambda)=\lambda^{2}+2 \lambda\left(\frac{\alpha}{\alpha+\beta}\right)+\frac{\alpha(\alpha+1)}{(\alpha+\beta)(\alpha+\beta+1)} \text {. }
$$

Now, if $\lambda=-\mu=\frac{-\alpha}{\alpha+\beta}$, , the second central moment becomes, from Equation 24,

$$
\begin{aligned}
& G_{2}\left(1 ; \frac{-\alpha}{\alpha+\beta}\right)=\left(\frac{-\alpha}{\alpha+\beta}\right)^{2}+2\left(\frac{-\alpha}{\alpha+\beta}\right)\left(\frac{\alpha}{\alpha+\beta}\right) \\
& +\frac{\alpha}{\alpha+\beta}\left(\frac{\alpha+1}{\alpha+\beta+1}\right)=\sigma^{2} .
\end{aligned}
$$

That is, the variance of the Beta distribution.

Suppose $\alpha=\beta=1$ in Equation 20 it yields the generalized moment generating function of the Uniform distribution as

$$
\begin{aligned}
G_{n}(c ; \lambda) & =\sum_{r=0}^{n}\left(\begin{array}{l}
n \\
r
\end{array}\right) \lambda^{n-r} \frac{\Gamma(c r+1)}{\Gamma(c r+2)} \\
& =\sum_{r=0}^{n}\left(\begin{array}{l}
n \\
r
\end{array}\right) \lambda^{n-r} \frac{1}{c r+1} .
\end{aligned}
$$

\subsection{The Gamma Family of Distributions}

Let $X$ be a gamma random variable with the density function:

$$
f(x)=\frac{1}{\beta^{\alpha} \Gamma(\alpha)} x^{\alpha-1} e^{-\frac{x}{\beta}} ; x \geq 0, \alpha>0, \beta>0 .
$$

Using Equation 3 in Equation 2,

$$
\begin{aligned}
& E\left(e^{t\left(x^{c}+\lambda\right)}\right) \\
& =\frac{t^{n}}{n !} \sum_{r=0}^{n}\left(\begin{array}{l}
n \\
r
\end{array}\right) \lambda^{n-r} \frac{1}{\beta^{\alpha} \Gamma(\alpha)} \int_{0}^{\infty} x^{c r+\alpha-1} e^{-\frac{x}{\beta}} d x .
\end{aligned}
$$

Letting $v=\frac{x}{\beta}$, integrating, simplifying and taking the coefficient of $\frac{t^{n}}{n !}$ yields

$$
G_{n}(c ; \lambda)=\sum_{r=0}^{n}\left(\begin{array}{l}
n \\
r
\end{array}\right) \lambda^{n-r} \frac{\beta^{c r} \Gamma(c r+\alpha)}{\Gamma(\alpha)} .
$$

All conceivable moments of the Gamma family of distributions are obtained using Equation 29.

For the second generalized moment of the $X$ where $X$ has the gamma distribution is

$$
\begin{aligned}
& G_{2}(c ; \lambda)=\lambda^{2}+2 \lambda \frac{\Gamma(c+\alpha) \beta^{c}}{\Gamma(\alpha)}+\frac{\Gamma(2 c+\alpha) \beta^{2 c}}{\Gamma(\alpha)} . \\
& \text { If } c=1 \text { then, } \\
& \qquad G_{2}(1 ; \lambda)=\lambda^{2}+2 \lambda \alpha \beta+\alpha(\alpha+1) \beta^{2} .
\end{aligned}
$$

Hence, if $\lambda=-\mu=-\alpha \beta$ where $\mu=\alpha \beta$ is the mean of the usual gamma distribution, then

$$
\begin{aligned}
& G_{2}(1 ;-\alpha \beta)=(-\alpha \beta)^{2}+2(-\alpha \beta)(\alpha \beta)+\alpha(\alpha+1) \beta^{2} \\
& =\alpha \beta^{2}=\sigma^{2} .
\end{aligned}
$$

That is, the variance of the usual gamma distribution.

The third generalized moment of the gamma family of distributions is obtained from Equation 29 as

$$
\begin{aligned}
G_{3}(c ; \lambda)= & \lambda^{3}+3 \lambda^{2} \beta^{c} \frac{\Gamma(c+\alpha)}{\Gamma(\alpha)} \\
& +3 \lambda \beta^{2 c} \frac{\Gamma(2 c+\alpha)}{\Gamma(\alpha)}+\beta^{3 c} \frac{\Gamma(3 c+\alpha)}{\Gamma(\alpha)} .
\end{aligned}
$$

If $c=1$ and $\lambda=-\alpha \beta$ where $\alpha \beta$ is the mean of the gamma distribution then

$$
G_{3}(1 ;-\alpha \beta)=2 \alpha \beta^{3} \text {. }
$$

Hence, the skewness of the gamma distribution is easily obtained as

$$
s k(1)=\frac{G_{3}(1 ;-\alpha \beta)}{\left(G_{2}(1 ;-\alpha \beta)\right)^{\frac{3}{2}}}=\frac{2}{\alpha^{\frac{1}{2}}} .
$$

In the same way, the fourth moment of the gamma distribution about the mean can be obtained as

$$
G_{4}(1 ;-\alpha \beta)=6 \alpha \beta^{4} .
$$

Hence, the kurtosis of the gamma distribution may be obtained as 


$$
k u(1)=\frac{G_{4}(1 ;-\alpha \beta)}{\left(G_{2}(1 ;-\alpha \beta)\right)^{2}}=\frac{6}{\alpha} .
$$

Suppose $\alpha=1$ in Equation 29, the generalized moment generating function of all forms of the exponential distribution is obtained as

$$
G_{n}(c ; \lambda)=\sum_{r=0}^{n}\left(\begin{array}{l}
n \\
r
\end{array}\right) \lambda^{n-r} \beta^{c r}(c r) \Gamma(c r) .
$$

Also, setting $\beta=2$ and $\alpha=\frac{k}{2}$ where $k=1,2, \ldots$ gives the generalized moment generating function of the chisquare distribution with $k$ degrees of freedom as

$$
G_{n}(c ; \lambda)=\sum_{r=0}^{n}\left(\begin{array}{l}
n \\
r
\end{array}\right) \lambda^{n-r} 2^{c r} \frac{\Gamma\left(c r+\frac{k}{2}\right)}{\Gamma\left(\frac{k}{2}\right)} .
$$

The generalized moment generating function can be used to obtain moments of powers of random variables with non-integer negative indices. For example, the gamma density in Equation 28; $\mathrm{cr}+\alpha>0$; that is, if the real number $c$ is such that $c \geq \frac{-\alpha}{\beta} ; r=1,2, \ldots$ and some specified value of $\alpha>0$. For instance, let $c=-\frac{3}{2}$ and $\alpha=5$.

Particularly, the possible moments of $X^{-\frac{3}{2}}$ are obtained using Equation 29. Thus,

$$
G_{1}\left(-\frac{3}{2} ; \lambda\right)=\lambda+2-\frac{3}{2} \frac{5 \sqrt{\pi}}{64} .
$$

Setting $\lambda=0$ in Equation 40 gives the mean of the distribution of $X^{-\frac{3}{2}}$ where $X$ has the gamma distribution as

$$
\mu=G_{1}\left(-\frac{3}{2} ; 0\right)=\frac{5 \sqrt{2 \pi}}{256} \text {. }
$$

Now, if $n=2 \quad c=1$ and $\lambda=-\frac{5 \sqrt{2 \pi}}{256}$ then the variance of $X^{-\frac{3}{2}}$ is obtained from Equation 30 as

$$
\begin{aligned}
& G_{2}\left(-\frac{3}{2} ; \frac{5 \sqrt{2 \pi}}{256}\right) \\
& =\frac{2^{-3}}{24}+\left(\frac{5 * 2^{-\frac{3}{2}} \sqrt{\pi}}{64}\right)^{2}-2\left(\frac{5 * 2^{-\frac{3}{2}} \sqrt{\pi}}{64}\right)^{2}=0.003 .
\end{aligned}
$$

\subsection{The Normal Distribution}

The generalized moment generating function of the random $X$ where $X$ has the normal distribution, with parameters $\mu$ and $\sigma^{2}$, and with $p d f$ given by

$$
f(x)=\frac{1}{\sigma \sqrt{2 \pi}} e^{-\left(\frac{x-\mu}{\sigma \sqrt{2}}\right)^{2}} ;-\infty<\mu<\infty ; \sigma^{2}>0
$$

may be obtained from Equation 1 as

$$
M_{\left(X^{c} ; \lambda\right)}(t)=\int_{-\infty}^{\infty} \sum_{n=0}^{\infty}\left(X^{c}+\lambda\right)^{n} \frac{t^{n}}{n !} \cdot \frac{e^{-\left(\frac{x-\mu}{\sigma \sqrt{2}}\right)^{2}}}{\sigma \sqrt{2 \pi}} d x
$$

By considering the coefficient of $\sum_{n=0}^{\infty} \frac{t^{n}}{n !}$ as the $n^{\text {th }}$ generalized moment of the distribution yields

$$
G_{n}(c ; \lambda)=\sum_{r=0}^{n}\left(\begin{array}{l}
n \\
r
\end{array}\right) \lambda^{n-r} \int_{-\infty}^{\infty} x^{c r} \cdot \frac{e^{-\left(\frac{x-\mu}{\sigma \sqrt{2}}\right)^{2}}}{\sigma \sqrt{2 \pi}} d x
$$

Letting $v=\left(\frac{x-\mu}{\sigma \sqrt{2}}\right)^{2}$, solving for $x$, expanding binomially integrating and simplifying gives

$$
\begin{aligned}
& G_{n}(c ; \lambda) \\
& =\sum_{r=0}^{n}\left(\begin{array}{l}
n \\
r
\end{array}\right) \lambda^{n-r} \sum_{t=0}^{c r}\left(\begin{array}{c}
c r \\
t
\end{array}\right) \mu^{c r-t}\left(2 \sigma^{2}\right)^{\frac{t}{2}} \frac{\Gamma\left(\frac{t}{2}+\frac{1}{2}\right)}{\sqrt{\pi}} .
\end{aligned}
$$

Equation 46 is evaluated at even values of $t$. Also, Equation 46 may be used to generate all conceivable moments of all forms of the normal distribution.

For instance, the second generalized moment of the random variable $X$ for $c=1$ where $X \sim N\left(\mu, \sigma^{2}\right)$ is

$$
\begin{aligned}
& G_{2}(1 ; \lambda)=\sum_{r=0}^{2}\left(\begin{array}{l}
2 \\
r
\end{array}\right) \lambda^{2-r} \sum_{t=0}^{r}\left(\begin{array}{l}
r \\
t
\end{array}\right) \mu^{r-t}\left(2 \sigma^{2}\right)^{\frac{t}{2}} \frac{\Gamma\left(\frac{t}{2}+\frac{1}{2}\right)}{\sqrt{\pi}} \\
& =\lambda^{2}+2 \lambda \mu+\mu^{2}+\sigma^{2} .
\end{aligned}
$$

Hence,

$$
G_{2}(1 ;-\mu)=\sigma^{2}
$$

Equation 48 is the variance of the normal distribution. Now,

$$
G_{3}(1 ; \lambda)=\lambda^{3}+3 \lambda^{2} \mu+3 \lambda \mu^{2}+3 \lambda \sigma^{2}+\mu^{3}+3 \mu \sigma^{2} .
$$

Hence,

$$
\begin{aligned}
G_{3}(1 ;-\mu)= & (-\mu)^{3}+3 \mu^{3}-3 \mu^{3} \\
& -3 \mu \sigma^{2}+\mu^{3}+3 \mu \sigma^{2}=0
\end{aligned}
$$

and

$$
\begin{aligned}
G_{4}(1 ; \lambda)= & \lambda^{4}+4 \lambda^{3} \mu+6 \lambda^{2}\left(\mu^{2}+\sigma^{2}\right) \\
& +4 \lambda\left(\mu^{3}+3 \mu \sigma^{2}\right)+\mu^{4}+6 \mu^{2} \sigma^{2}+3 \sigma^{2} .
\end{aligned}
$$

Hence,

$$
G_{4}(1 ;-\mu)=3 \sigma^{4}
$$


Now, the skewness of the normal distribution may be obtained as

$$
\operatorname{sk}(1)=\frac{G_{3}(1 ;-\mu)}{\left(G_{2}(1 ;-\mu)\right)^{\frac{3}{2}}}=0
$$

implying that the distribution is symmetric (Arua et al 1997).

The kurtosis of the normal distribution may be obtained as

$$
k u(1)=\frac{G_{4}(1 ;-\mu)}{\left(G_{2}(1 ;-\mu)\right)^{2}}=\frac{3 \sigma^{4}}{\left(\sigma^{2}\right)^{2}}=3
$$

implying a mesokurtic distribution [6].

\section{Conclusion}

This paper has developed and presented the generalized moment generating functions of random variables and their probability distributions. The method has been shown to be quicker and easier to apply than the traditional moment generating functions which may not exist for some distributions. Thus, the generalized moment generating function is more versatile than the traditional moment generating function. The new method was illustrated with a general probability distribution function, the beta family of distributions, the gamma family of distributions and the normal distribution. However, this method has not been developed for discrete probability distributions.

\section{Competing Interest}

The authors have no competing interest.

\section{List of Abbreviations}

$G_{x}(t)$ is the generalized moment generating function of the random variable, $X$.

$G_{n}(c ; \lambda)$ is the $n^{\text {th }}$ moment of the $c^{\text {th }}$ power of the random variable, $X$ about an arbitrarily chosen constant, $\lambda$.

\section{References}

[1] Onyeka, A. (2000). Probability Distributions. K. K. Integrated Nigeria. Pp. 22 and 43.

[2] Oyeka, I. C. A.; Ebuh G. U.; Nwosu, C. R.; Utazi, E. C.; Ikpegbu, P. A.; Obiora-Ilouno, H and Nwankwo, C. C. (2010). 'Moment Generating Function of $\mathrm{X}^{\mathrm{C}}$.' Global Journal of Mathematics and Statistics, India. Volume 3, Number 1, 2010.

[3] Oyeka, I. C. A.; Onyediakachi, I. P.; Ebuh, G. U.; Utazi, C. E.; Nwosu, C. R.; Obiora-Ilouno, H and Nwankwo, C. C. (2012). 'Moment Generating Function of $\mathrm{X}^{\mathrm{c}}, \mathrm{Y}^{\mathrm{d}}$.' African Journal of Mathematics and Computer Science Research. Volume 5 (14). Pp. 247-252.

[4] Feller, W. (1966. An Introduction to Probability Theory and Applications. Volume II. Second Edition. John Wiley and Sons, Inc. New York. Pp. 133, 472-473.

[5] Grimmett, G. and Welsh, P. (1986). Probability. An Introduction. Oxford University Press, USA. Pp. 101.

[6] Arua, A. I.; Chukwu, W. I. E.; Okafor, F. C. \& Ugwuowo, F. I. (1997). Fundamentals of Statsitics for Higher Education. Fijac Academic Press, Nsukka, Nigeria. P 33 \& 63. 